\title{
A cystic lesion of the adrenal gland mimicking hydatid cyst
}

Faten Hadj Kacem (1), Mouna Ammar (1), Ayman Maalej (2), Nesrine Cheikhrouhou (1), Nadia Charfi ( 1), Mohamed Abid (1)

(1) Department of Endocrinology, sfax, Tunisia

(2) Imaging Department, sfax, Tunisia

\section{Introduction:}

Adrenal cystic lesions are uncommon and mostly identified incidentally during radiological investigations or at surgery for unrelated reasons.

Its incidence varies between 0,064 and $0,18 \%$.

Adrenal cysts are classified as endothelial cysts (45\%), pseudocysts (39\%), epithelial cysts (9\%), and parasitic cysts $(7 \%)$.

\section{Case Report:}

A 45-year-old woman with a past medical history of systemic hypertensive disease for 2 years, presented with recurrent abdominal pain during the last 3 months.

Clinical examination was normal with stabile hypertension.

Abdominal ultrasonography (US) showed a multi-cystic mass in the right adrenal region.

The CT scan revealed a hypo-dense, multicystic mass measuring $6 \times 5 \mathrm{~cm}$ in the right adrenal bed, with slightly mural enhancing after administered with contrast medium and calcifications of the cystic wall. The pictures obtained were very evocative of hydatid cyst (Figure 1 ).

Hydatid serology was negative.

An adrenal evaluation was performed and was within normal limits.

On the basis of these findings, surgical excision was carried out with a subcostal flank incision, grossly showing an $8 \times 7 \mathrm{~cm}$ well-defined, multiloculated cystic lesion.

The cyst was excised and a right adrenalectomy was perfomed without any intraoperative complications.

Histologically, the cyst showed a multicystic architecture with dilated spaces lined with flattened, simple endothelial cells. The cystic spaces often contained calcifications.

There were no hydatid membranes and no evidence of malignance was identified in the adrenal mass.

The histopathological diagnosis was a cystic lymphangioma in the right adrenal gland. The patient's postoperative course was uneventful. Three months after the operation, she did not have abdominal pain and she experienced no signs or symptoms of endocrine dysfunction.
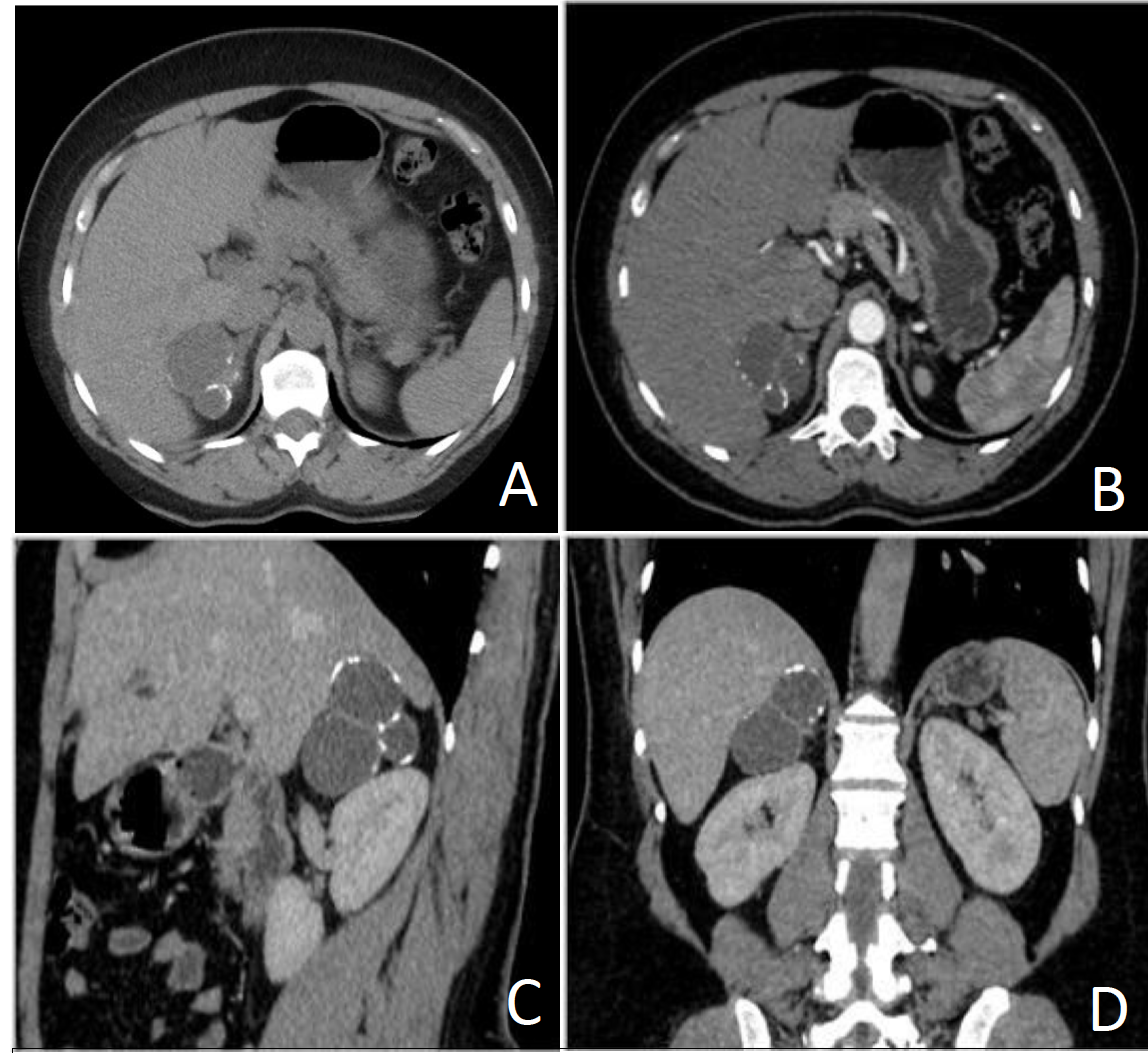
$5 * 6 \mathrm{~cm}$ with calcifications in the cystic wall; axial section.

(B, C, D) Post-contrast CT scan demonstrates a non-enhancing lesion; axial, sagittal and coronal sections.

\section{Discussion:}

Adrenal cystic lymphangioma ( $\mathrm{ACL}$ ) is a very rare benign vascular lesion and a subtype of endothelial adrenal cysts. Cystic lymphangioma developing in the adrenal gland is extremely rare. The first case of ACL had been reported in 1965, recently less than 50 immunohistochemically proven cases have been reported in the literature (1).

The ACL in our case affected female patient and arise in the right-sided adrenal as seen in Ellis et al's observations that ACL had a significant female predisposition and twice more frequently involved right-sided adrenal (1) .

ACLs are usually asymptomatic. However, symptoms can occasionally occur and they are typically related to the overall mass of the cyst .The most common symptoms are abdominal or flank pain, gastrointestinal discomfort, palpable mass and arterial hypertension and palpitation especially in functional cysts $(2,3)$.

On ultrasound (US), ACL is a well-marginated, anechoic lesion typically located on the suprarenal area and which can show shadows and internal echoes, when calcifications or internal debris are present $(1,4)$. On CT scan the mass is identified as a hypodense, non-enhanced lesion with smooth borders and thin wall $(<3,5 \mathrm{~mm})$ . US and CT scans can reveal peripheral calcifications of the cystic wall in 15\% of cases. Such calcifications in the cystic wall were observed in our case.

The differential diagnosis of $\mathrm{ACL}$ includes cystic benign or malignant adrenocortical neoplasms as well as other cyst types. Pseudocysts represent the most common type of adrenal cyst and have been thought to be a result of hemorrhage within a tumor or hemorrhage into adrenal parenchyma due to trauma, toxic or infectious process(1). Hydatid cyst of the adrenal gland is exceptional and accounts for only $6 \%$ to $7 \%$ of all adrenal cysts but it should be considered in endemic areas(5)

\section{Conclusion:}

$\mathrm{ACL}$ is a very rare entity. Most of the ACLs reported are asymptomatic but symptoms can occasionally occur and they are typically related to the overall mass of the cyst. Imaging can characterize the cystic nature of these lesions. However, it sometimes fails to establish a specific diagnosis preoperatively. So definite diagnosis relies on Histologic and immunohistochemical examination.

Lymphangiomas must be kept in mind in the clinical and radiologic differential diagnosis of cystic adrenal lesions.

\section{References:}

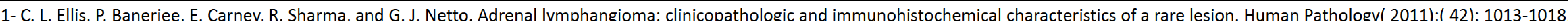

2- L. A. Erickson, R. V. Lloyd, R. Hartman, and G. Thompson. Cystic adrenal neoplasms. Cancer. (2004);(101): 1537-1544.

3- Y. Tanuma, M. Kimura, and S. Sakai. Adrenal cyst: a review of the japanese literature and report of a case. International Journal of Urology. (2001); (8): 500-503.

4- J.M. Longo, S.Z. Jafri, andK. B. Bis. Adrenal lymphangioma: acase report. Clinical Imaging. (2000);(24): 104-106.

5- Goel MC, Agarwal MR, Misra A. Percutaneous drainage of renal hydatid cyst: early results and follow-up. Br J Urol. (1995); (75):724-8. 